\title{
Addendum to "The Effect of Medical Marijuana Laws on Marijuana-Related Attitude and Perception among US Adolescents and Young Adults"
}

\author{
H. Wen ${ }^{1}$ • J. M. Hockenberry ${ }^{2}$ • B. G. Druss ${ }^{2}$ \\ Published online: 15 February 2019 \\ (C) Society for Prevention Research 2019
}

Since the publication of our paper, entitled "The Effect of Medical Marijuana Laws on Marijuana-Related Attitude and Perception among U.S. Adolescents and Young Adults" (Wen et al. 2019), we have become aware of a related paper, which was previously published by Schmidt and colleagues, entitled "Young People's More Permissive Views About Marijuana: Local Impact of State Laws or National Trend?" (Schmidt et al. 2016). We appreciate the opportunity to correct this omission and to highlight some of the differences between the two studies.

Although both studies used restricted-access data from the National Survey on Drug Use and Health (NSDUH), differences in the approaches accounted for our divergent findings. First, we created the risk perception question differently, dichotomizing between "low or no risk" and "moderate to great risk" instead of dichotomizing between "great risk" versus all others. Second, while both papers used a quasi-experimental difference-in-differences design operationalized through twoway fixed effects to estimate the policy effect net of the potential confounding effect of the time-invariant state heterogeneity and national secular trend in marijuana-related attitudes and perceptions, our work differed in that we also included state-specific linear time trends. This approach not only accounted for the evolving social norms and sentiments, but also allowed for state-specific differences in those trajectories. By doing so, we found that state implementation of medical marijuana laws was associated with an increase in the

H. Wen

hefei.wen@uky.edu

1 Department of Health Management \& Policy, University of Kentucky College of Public Health, 111 Washington Avenue, CPH 103C, Lexington, KY, USA

2 Department of Health Policy \& Management, Emory University Rollins School of Public Health, 1518 Clifton Road NE., Atlanta, GA, USA probability that young adults perceived no/low health risk related to marijuana use and a decrease in the probability that adolescents perceived parental acceptance of marijuana use. Our findings differed from the Schmidt and colleagues' study suggesting that "there is a national trend toward young people taking more permissive views about marijuana independent of any effects within states." (Schmidt et al. 2016, pp.1498)

\section{Compliance with Ethical Standards}

Conflict of Interest The authors declare that they have no conflict of interest.

Publisher's Note Springer Nature remains neutral with regard to jurisdictional claims in published maps and institutional affiliations.

\section{References}

Schmidt, L. A., Jacobs, L. M., \& Spetz, J. (2016). Young people's more permissive views about marijuana: Local impact of state laws or national trend? American Journal of Public Health, 106(8), 14981503.

Wen, H., Hockenberry, J. M., \& Druss, B. G. (2019). The effect of medical marijuana laws on marijuana-related attitude and perception among US adolescents and young adults. Prevention Science. https://doi.org/10.1007/s11121-018-0903-8. 